\title{
PRIME IDEAL STRUCTURE IN COMMUTATIVE RINGS $\left(^{(}\right)$
}

\author{
BY \\ M. HOCHSTER
}

0. Introduction. Let $\mathscr{C}$ be the category of commutative rings with unit, and regard $\operatorname{Spec}($ as in [1]) as a contravariant functor from $\mathscr{C}$ to $\mathscr{T}$, the category of topological spaces and continuous maps. The spaces of the form $\operatorname{Spec} A, A$ a ring (ring always means object of $\mathscr{C}$ ), are well known to have many special properties. It is worthwhile to discover whether these well-known properties of the spaces characterize them, since we then will know the limitations of the topological approach. As a by-product of this study, remarkable facts about the structure of the prime ideals in a commutative ring come to light. E.g. given any ring $A$ there is a ring whose prime ideals have precisely the reverse order of the primes of $A$. In the same vein, on the topological level there is a complete duality between localization and taking residue class rings (see $\S 8$, Proposition 8 ).

We call a space spectral if it is $T_{0}$ and quasi-compact, the quasi-compact open subsets are closed under finite intersection and form an open basis, and every nonempty irreducible closed subset has a generic point. Spec $A, A$ a ring, is well known to be spectral, and in fact these properties do characterize the spaces in the image of Spec. However, there are much more enlightening characterizations. For example, the last property on the list is a very special case of a much more general property of spectral spaces-best described as the compactness of a new topology on the spectral space which is derived from the original topology. Again, the spectral spaces are precisely the projective limits of finite $T_{0}$ spaces.

Proving that these properties characterize the image of Spec involves constructing a large number of rings. This construction $(\$ \S 3-7)$ is very intricate, but is practically choice-free and turns out to be fairly functorial. Moreover, we can fix any field $k$ and get the constructed rings to be $k$-algebras.

To be more precise about the functorial aspects, we define a continuous map of spectral spaces to be spectral if inverse images of quasi-compact open sets are quasi-compact. Let $\mathscr{S}$ be the subcategory of $\mathscr{T}$ consisting of spectral spaces and spectral maps. Then it is well known that every space and map in the image of Spec is in $\mathscr{S}$ and it will turn out that up to isomorphism, every space and map in $\mathscr{S}$ is in the image of Spec. In this sense, we may say that $\mathscr{S}$ is precisely the image of Spec.

We say that Spec is invertible on a subcategory $\mathscr{R}$ of $\mathscr{S}$ if there is a (contravariant) functor $F: \mathscr{R} \rightarrow \mathscr{C}$ whose composition with Spec is isomorphic with the inclusion

Received by the editors August 5, 1968.

( ${ }^{1}$ ) This research was partially supported by NSF grant GP-8496. 
functor of $\mathscr{R}$ in $\mathscr{S}$; also, we shall say that $F$ is a space-preserving functor from $\mathscr{R}$ to $\mathscr{C}$.

It turns out that Spec can be inverted on surprisingly large subcategories of $\mathscr{S}$. At this point, the results of $\S 7$ should be read as part of this introduction. In fact, after looking at $\S 1, \S 2$ and the results of $\S 7$, the reader can continue and finish the paper (except for the proof of Theorem 9 in $\$ 16$ ), and then go back to the technical $\S \S 3-6$ if he wishes.

We note that $\$ 16$ uses the functorial nature of our constructions to characterize the underlying spaces of preschemes and schemes.

Most of the results of this paper were obtained in the author's doctoral thesis [4]. The author wishes to thank once more his advisor, Professor G. Shimura. Another part of the thesis, greatly generalized, will appear separately [5].

1. The technique for inverting Spec. The main purpose of this section is to put into perspective the constructions and results of the technical $\S 3-6$, where the machinery needed for building functors which invert $\mathrm{Spec}$ on various subcategories of $\mathscr{S}$ is developed. However, we also outline or mention briefly most of the other sections.

$\$ 2$ contains certain topological material which is needed throughout. The main results may be summarized thus: the spectral subobjects (subspaces whose inclusions are spectral maps) of a spectral space $X$ form the closed sets of a compact ( $\equiv$ quasi-compact Hausdorff) topology for $X$. An open sub-basis for this topology consists of the quasi-compact open subsets of $X$ and their complements. (We note that R. G. Montgomery has recently been investigating, quite independently, this topology on ring spectra for application to the study of rings of continuous runctions in his developing thesis at Clark University. See also [8].) A corollary is that a spectral subobject of a spectral space is closed in the original topology iff it contains the closure of each of its points, a result very much needed for our constructions.

$\$ \S 3-6$, as mentioned, are rather technical. The basic idea is this: if a ring having a given spectrum $X$ can be found, then a reduced such ring can be found. But a reduced ring can be represented as a ring of functions on its spectrum, with the values at a given point being taken in the residue class domain at that point. We partially axiomatize this situation in $\S 3$, and arrive at the notion of a spring. Not every spring comes from a ring; those that do, we call affine. $\S 4$ introduces the concept of an indexed spring. The index is an extra structure needed so that we will be able to modify a given spring into an affine spring. Intuitively, if we had a ring $A$ rather than merely a spring, two primes $P \subset Q$ of $A$, and an element $a \in Q / P$, the index would measure the "order" to which $a$ vanishes at $Q / P$. E.g. if the localization $B$ of $A / P$ at $Q / P$ were a discrete valuation ring, this "order" might naturally be taken to be the integer assigned to $a$ by the valuation. In the precise formulation of $\S 4$, the index is actually a family of valuations indexed by the pairs of points $(y, x)$ of the underlying space such that $x \in \mathrm{Cl} y$. 
$\S 4$ also develops a method of converting indexed springs into affine springs, provided one additional condition, treated in $\$ 5$, is satisfied. $\$ 6$ describes a process for constructing indexed springs given, roughly speaking, a space and a family of indeterminates. At every stage we work with a category, not merely with objects.

$\$ 7$ combines the results of the previous sections to invert Spec on various subcategories of $\mathscr{S}$. The question of whether the results obtained are optimal is also considered.

The remaining $\$ \S 8-16$ deal with miscellaneous related topics: more properties of spectral spaces, the category $\mathscr{S}$ and a certain extension of it, category operations, a certain class of ring epimorphisms, maximal ideal spaces, the order of the primes in a ring, and the underlying spaces of preschemes and schemes. The titles of the sections should be sufficiently explanatory.

2. Patches. Let $X$ be a spectral space. By the patch topology on $X$ we mean the topology which has as a sub-basis for its closed sets the closed sets and quasicompact open sets of the original space (or better, which has the quasi-compact open sets and their complements as an open sub-basis). By a patch in $X$ we mean a set closed in the patch topology.

THEOREM 1. The patch topology on $X$ is compact.

Proof. It is certainly Hausdorff. Quasi-compactness will follow if every family of closed and quasi-compact open sets maximal with respect to having the finite intersection property intersects. But it is not difficult to see that the intersection of all the closed sets in such a family must also be in the family, and that it must be irreducible. Its generic point is then in the intersection.

We indicate a few more results along these lines. The proofs are straightforward and are omitted. If $Y$ is a spectral subobject of $X$, the topology $Y$ inherits when $X$ is given the patch topology is the same as the patch topology for $Y$. If $X^{\prime}$ is another spectral space then $f: X \rightarrow X^{\prime}$ is spectral iff it is continuous in both the original and patch topologies. Moreover $f(X)$ is a patch in $X^{\prime}$. It then follows that $Y$ is a spectral subobject of $X$ iff $Y$ is a patch in $X$.

COROllary. If $Y$ is a patch in $X, x$ is in $\mathrm{Cl} Y$ iff it is in the closure of some point of $Y$.

This is proved by using Theorem 1 to show that there is a point in the intersection of $Y$ and all the quasi-compact $X$-open neighborhoods of $x$. Similarly:

COROLlary. Any two points of $X$ either have disjoint open neighborhoods or are in the closure of a third point.

3. Springs. We shall define several categories with natural forgetful functors to $\mathscr{S}$, and some functors between these categories. In each case it will be obvious that these functors commute with the forgetful functors up to isomorphism of functors. We first consider the category $\mathscr{A}$ whose objects are the pairs $(X, A)$, 
where $A$ is a ring without radical and $X$ is a dense patch in Spec A, and whose morphisms are just the ring homomorphisms $h$ such that Spec $h$ carries the one given patch into the other, but with the ring homomorphisms in the opposite direction from that of arrows in $\mathscr{A}$. An object $(X, A)$ of $\mathscr{A}$ will be called affine if $X=\operatorname{Spec} A$. We observe that from each object $(X, A)$ of $\mathscr{A}$ we obtain a triple $\left(X,\left\{A_{x}\right\}, A\right)$, where $A_{x}=A / x, x$ regarded as an ideal. Here $X$ is a spectral space, $\left\{A_{x}\right\}$ is a family of domains indexed by $X$, and $A$ is a subring of $\prod_{x \in X} A_{x}$ ( $A$ is without radical). In fact, given such a triple, it comes from an (essentially unique) object in $\mathscr{A}$ iff the following conditions hold:

(1) For all $x$ in $X, A_{x}=\{a(x): a \in A\}$.

(2) For all $a \in A, d(a)=\{x \in X: a(x)=0\}$ is quasi-compact and $X$-open.

(3) $\{d(a): a \in A\}$ is an open basis for $X$.

(We also introduce the notation $z(a)$ for $X-d(a)$.)

The proof is straightforward. We note only that given such a triple, the required embedding $\phi$ of $X$ into Spec A is given by $x \mapsto\{a \in A: a(x)=0\}$. Thus, we alternatively regard the objects of $\mathscr{A}$ as such triples. One may phrase the definition of morphism in $\mathscr{A}$ directly in terms of these triples as follows: a morphism is a pair $(f, h)$, where $f$ is a map of the underlying spectral spaces (same direction) and $h$ is a homomorphism of the rings (opposite direction) such that for each $a$, $f^{-1}(d(a))=d(h(a))$ (equivalently, $\left.f^{-1}(z(a))=z(h(a))\right)$.

Strictly speaking, these triples and their morphisms form a category merely equivalent to $\mathscr{A}$; it is desirable however to speak loosely and make the identification. We shall refer to the objects and morphisms of $\mathscr{A}$ as springs and spring morphisms. In a hypothesis, $A$ will always represent the spring $(X, A)$ or $\left(X,\left\{A_{x}\right\}\right.$, $A$ ); we make a similar convention for $\boldsymbol{A}^{i}, \boldsymbol{A}^{\prime}$, etc.

One more bit of notation. In discussing a ring $A$, we write $D(a)$ for $\{x \in \operatorname{Spec} A: a$ $\notin x\}$ and $Z(a)$ for Spec $A-D(a)$. If $B$ is any subset of $A$, we write $R_{A}(B)$ (or sometimes simply $R(B)$ ) for the intersection of the prime ideals of $A$ containing $B$, or, equivalently, for the ideal of elements with some power in the ideal $(B)$ generated by $B$.

THEOREM 2. A spring $A$ is affine if and only if:

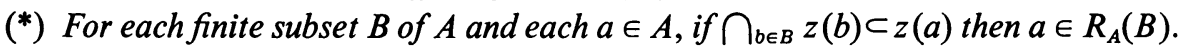

Proof. (*) holds trivially if $\boldsymbol{A}$ is affine. Assume $\left(^{*}\right)$. We want to show that $\phi(X)$ $=\operatorname{Spec} A$. If not, since $\phi(X)$ is a patch of Spec $A$ there is a finite subset $B$ of $A$ and an $a \in A$ such that $\phi(X) \subset U_{a, B}=Z(a) \cup\left(\bigcup_{b \in B} D(b)\right)$ and $U_{a, B} \neq \operatorname{Spec} A$, for the sets $U_{a, B}$ are a closed basis for the patch topology on Spec $A$. But $\phi(x) \subset U_{a, B}$ and (*) imply $a \in R_{A}(B)$, from which $U_{a, B}=\operatorname{Spec} A$, the required contradiction.

We shall find it not difficult to construct springs, but much harder to get them to satisfy (*). We do this by taking extensions, in the following sense: if $\boldsymbol{A}, \boldsymbol{A}^{\prime}$ are springs and $X=X^{\prime}$, we say that $A^{\prime}$ extends $A$ if for each $x$ in $X, A_{x}^{\prime}$ is a subring of $A_{x}^{*}$ and contains $A_{x}$. Here, if $B$ is any domain, $B^{*}$ denotes the field of fractions of 
$B$. We make a couple of definitions more in this direction. If $A$ is a spring and $B$ a subset of $\prod_{x \in X} A_{x}^{*}$ such that for each $c \in C=A[B]$ (the subring of $\prod_{x \in X} B_{x}^{*}$ generated by $A$ and $B) d(c)$ is quasi-compact open, then $C=(X,\{\{c(x): c \in C\}\}, C)$ is a spring extending $A$, and we write $C=A[B]$. We say $B$ induces the extension $C$.

We add one more definition. If $\boldsymbol{A}$ is a spring and $a, b \in A$ are such that $z(b)$ $\subset z(a)$, then $a \# b \in \prod_{x \in X} A_{x}^{*}$ is defined as having the value 0 or $a(x) / b(x)$, according as $x \in z(b)$ or $d(b)$.

4. Indices. If $X$ is a spectral space, let $\sigma(X)$ be the subset of pairs $(y, x)$ such that $x \in \mathrm{Cl} y$. If $f: X \rightarrow X^{\prime}$ is spectral, there is an induced map (which we also call $f$ ) from $\sigma(X)$ to $\sigma\left(X^{\prime}\right)$ given by $f(y, x)=(f(y), f(x))$. By an index $v$ on a spring $A$ we mean a function which assigns to each pair $p=(y, x) \in \sigma(X)$ an additive valuation $v_{p}$ from $A_{y}^{*}$ to the integers in such a way that:

$\left(\mathrm{I}_{1}\right)$ For all $a \in A$ and $p=(y, x) \in \sigma(X)$ such that $y \in d(a)$ we have $0 \leqq v_{p}(a)$ with equality if and only if $a(x) \neq 0$; here, we have written $v_{p}(a)$ for $v_{p}(a(y))$, a practice we shall follow frequently since there is no danger of confusion.

$\left(\mathrm{I}_{2}\right)$ For all $a \in A$ there is an integer $N>0$ such that if $p=(y, x) \in \sigma(X)$ and $y \in d(a)$, then $v_{p}(A) \leqq N$.

A spring together with an index for it will be called an indexed spring. By a morphism of indexed springs we mean a spring morphism $(f, h)$ from say $A, v$ to $A^{\prime}, v^{\prime}$ such that for each $a \in A^{\prime}$ and $p=(y, x) \in \sigma(X)$ with $y \in d(h(a))$, we have $v_{p}(h(a))=v_{f(p)}(a)$. We have thus defined a category, which we call $\mathscr{B}$.

If $A, v$ is an indexed spring and $\boldsymbol{A}^{\prime}$ is an extension of $\boldsymbol{A}$ such that $v$ is also an index for $\boldsymbol{A}^{\prime}$, we call $\boldsymbol{A}^{\prime}$ a $\boldsymbol{v}$-extension of $\boldsymbol{A}$.

The idea behind introducing indices is that we want to take fairly arbitrary springs and modify them so that $\left({ }^{*}\right)$ holds, i.e. so that they are affine. It is natural to try to do this by taking extensions. E.g. if $\boldsymbol{A}$ is an affine spring it must be true that if $a, b \in A$ and $z(b) \subset z(a)$ then for some integer $n, a^{n} \# b \in A$. However, for an arbitrary spring $\boldsymbol{A}$ it need not be true that $a^{n} \# b$ induces an extension, no matter how large we take $n$. There is nothing to guarantee that zero sets of polynomials in $a^{n} \# b$ will be closed. The index remedies this situation perfectly.

THEOREM 3. Let $\boldsymbol{A}, v$ be an indexed spring, and let $a, b \in A$ be such that $z(b) \subset z(a)$, so that $a \# b$ is defined. Then the following conditions on the pair $(a, b)$ are equivalent.

(i) For each $p=(y, x) \in \sigma(X)$ with $y \in d(a), v_{p}(A) \geqq v_{p}(b)$, with equality only if $x \in d(b)$.

(ii) $a \# b$ induces a $v$-extension of $\boldsymbol{A}, v$.

Proof. (ii) $\Rightarrow$ (i) is straightforward. Assume (i). We first show that $a \# b$ induces an extension on $\boldsymbol{A}, \boldsymbol{v}$. Let $t$ be an indeterminate over $A$. We must prove that for any $q=\sum_{i} a_{i} t^{i}$ in $A[t], 0 \leqq i \leqq m, d(r)$ is quasi-compact open, where $r=q(a \# b)$. Let $c=b^{m} r=\sum_{i} a_{i} a^{i} b^{m-i} . c$ is in $A$. Now $d(r)$ is a patch, for

$$
d(r)=(d(c) \cap d(a)) \cup\left(d\left(a_{0}\right) \cap z(a)\right) .
$$


Thus, it remains to show that $z(r)$ is closed. We use two representations of $z(r)$ :

(1) $z(r)=(z(c) \cap d(b)) \cup\left(z\left(a_{0}\right) \cap z(b)\right)$ and

(2) $z(r)=(z(c) \cap d(a)) \cup\left(z\left(a_{0}\right) \cap z(a)\right)$.

It is easy to see that (1) and (2) hold. Hence, $z(r)$ is a patch. Then to show $z(r)$ closed it suffices to show that if $y \in z(r)$ and $x \in \mathrm{Cl} y$ then $x \in z(r)$. Since $z\left(a_{0}\right)$ $\cap z(a)$ is closed in $X$, we may assume, from (2), that $y \in z(c) \cap d(a)$. Since $z(c)$ $\cap d(b)$ is closed in $d(b)$, we may assume, from (1), that $x \in z(b)$. We can now apply i) to conclude that $v_{p}(a)>v_{p}(b)$, where $p=(y, x)$. Then $r(y)=0$ yields $-b(y)^{m} a_{0}(y)$ $=\sum_{i>0} a_{i}(y) a(y)^{i} b(y)^{m-i}$. But $v_{p}\left(-b^{m} a_{0}\right)=m v_{p}(b)+v_{p}\left(a_{0}\right)$, while $v_{p}\left(\sum_{i>0} a_{i} a^{i} b^{m-i}\right)$ $>m v_{p}(b)$ by (i) and the standard properties of valuations. Then $v_{p}\left(a_{0}\right)>0 \Rightarrow a_{0}(x)$ $=0 \Rightarrow x \in z\left(a_{0}\right)$. Thus, $x \in z\left(a_{0}\right) \cap z(b) \subset z(r)$, as required.

The verification that $v$ is an index for this induced extension is straightforward, and we omit it.

Now, if $A, v$ is an indexed spring, let $G(A, v)$ be the set of pairs $(a, b)$ of elements of $A$ such that $z(b) \subset z(a)$ and $a \# b$ induces a $v$-extension of $A$. From the previous theorem, $(a, b) \in G(A, v)$ iff $z(b) \subset z(c)$ and condition (i) holds. This condition, unlike, a priori, the defining condition of $G(A, v)$, does not depend on whether we look at the pair in $\boldsymbol{A}$ itself or some $v$-extension of $\boldsymbol{A}$. In other words, if $\boldsymbol{A}^{\prime}$ is a $v$-extension of $A$, then $G\left(A^{\prime}, v\right) \cap(A \times A)=G(A, v)$. As an immediate corollary, each finite subset of $G(A, v)$ induces a $v$-extension of $A$, and hence it easily follows that $G(A, v)$ induces a $v$-extension $\boldsymbol{A}^{1}$ of $\boldsymbol{A}$. Inductively then, if $\boldsymbol{A}^{n}$, a $v$-extension of $A$, has been defined, let $A^{n+1}$ be the $v$-extension of $A^{n}$ induced by $G\left(A^{n}, v\right)$. Finally, let $M(A)$ (properly, $M(A, v)$ ) be the $v$-extension of $A$ induced by the union of the underlying rings of all the $\boldsymbol{A}^{n} . M(A)$ is the closure of $\boldsymbol{A}$ under the operation of adjoining $a \# b$ whenever possible (i.e. whenever a $v$-extension is induced).

Proposition 1. Let $A, v$ be an indexed spring. Then $M(A)$ has the following property:

$\left({ }^{* *}\right)$ For all $a, b$ in the underlying ring of $M(A)$, if $z(b) \subset z(a)$ then $a \in R(b)$.

Proof. Choose $n$ so large that $a, b$ are in the underlying ring of $\boldsymbol{A}^{n}$. Choose an integer $N>0$ such that for $p=(y, x) \in \sigma(X)$ and $y \in d(b), v_{p}(b) \leqq N$. It is easy to check that $\left(a^{N+1}, b\right) \in G\left(A^{n}, v\right)$. Hence, $a^{N+1} \# b$ is in the underlying ring of $A^{n+1}$, and therefore in the underlying ring of $M(A)$.

We shall soon develop a condition under which $\left(^{* *}\right) \Rightarrow\left({ }^{*}\right)$, and which $A$ and $M(A)$ always satisfy alike. The problem of constructing a ring with a given spectrum $X$ will then reduce to the problem of constructing an indexed spring $A$, satisfying this condition, with $X$ as underlying space: for then, $\operatorname{Spec} M(A)$ will be homeomorphic with $X$.

Before we head in this direction, we show how $M$ can be made into a functor, i.e. defined on morphisms. In fact, the following proposition shows that there is an obvious unique way of defining $M$ on morphisms. The proof, which is not at all difficult, is omitted. The technique is like that used in proving Theorem 3. 
Proposition 2. Let $(f, h)$ be an indexed spring morphism from $A, v$ to $\boldsymbol{A}^{\prime}, v^{\prime}$. Let $(a, b) \in G\left(A^{\prime}, v^{\prime}\right)$. Then $(h(a), h(b)) \in G(A, v)$ and there is a unique extension $h_{1}$ of $h$ to a homomorphism from $A^{\prime}[a \# b]$ to $A[h(a) \# h(b)]$ such that $h_{1}(a \# b)=h(a) \# h(b)$, and for this $h_{1},\left(f, h_{1}\right)$ is an indexed spring morphism from $A[h(a) \# h(b)], v$ to $\boldsymbol{A}^{\prime}[a \# b], v^{\prime}$. Moreover if $\boldsymbol{A}^{\prime \prime}, \boldsymbol{A}^{\prime \prime \prime}$ extend $\boldsymbol{A}, \boldsymbol{A}^{\prime}[a \# b]$ respectively and $h_{2}: A^{m} \rightarrow A^{\prime \prime}$ is a homomorphism agreeing with $h$ on $A^{\prime}$ such that $\left(f, h_{2}\right)$ is a spring morphism from $A^{\prime \prime}$ to $\boldsymbol{A}^{\prime \prime}$, then $h(a) \# h(b) \in A^{\prime \prime}$ and $h_{2}(a \# b)=h(a) \# h(b)$.

It is clear that given an indexed spring morphism $\boldsymbol{A}, \boldsymbol{v} \rightarrow \boldsymbol{A}^{\prime}, v^{\prime}$ it has a unique natural extension $A^{1}, v \rightarrow A^{\prime 1}, v^{\prime}$, whence, inductively, a natural extension $A^{n}, v$ $\rightarrow A^{\prime n}, v^{\prime}$ for each $n$, and therefore an extension $M(A), v \rightarrow M\left(A^{\prime}\right), v^{\prime}$. Henceforth, we regard $M$ as a functor. Note that $M$ does not change underlying spaces or maps of underlying spaces; we say that $M$ is space-preserving.

5. Simple springs. If $A$ is a spring such that all the domains $A_{x}$ are subrings of a single ring $K$, so that $A$ may be regarded as a subring of the ring of functions from $X$ to $K$, we say that $A$ is simple if the image of each element of $A$ in $K$ is finite. $\boldsymbol{A}, \boldsymbol{v}$ is called simple if $\boldsymbol{A}$ is.

THEOREM 4. If $\boldsymbol{A}, v$ is simple, so is $M(\boldsymbol{A})$. If $\boldsymbol{A}$ is simple and (**) holds, then (*) holds, so that $\boldsymbol{A}$ is affine. If $\boldsymbol{A}, v$ is simple, then $M(A)$ is affine.

Proof. If $a, b$ have finite images, so does $a \# b$, whence the first statement is clear. The remaining statements follow from the second. Comparing $\left({ }^{* *}\right)$ and $\left({ }^{*}\right)$, we see that it will be enough to show that if $B$ is a finite subset of $A$, then there is an element $c$ in the ideal of $A$ generated by $B$ such that $z(c)=\bigcap_{b \in B} z(b)$. By a trivial induction, we can reduce to the case where $B=\left\{b_{1}, b_{2}\right\}$ has two elements. Taking complements, we want $c \in\left(b_{1}, b_{2}\right)$ such that $d(c)=d\left(b_{1}\right) \cup d\left(b_{2}\right)$. Let $W_{j}(1), \ldots, W_{j}\left(m_{j}\right), j=1,2$, be the subsets of $d\left(b_{j}\right)$ on which $b_{j}$ is constant. Choose a point $y_{W}$ in each $W$, and also a point $y\left(W_{1}, W_{2}\right)$ in each nonempty intersection of a $W_{1}$ and a $W_{2}$. Let $Y \subset d\left(b_{1}\right) \cup d\left(b_{2}\right)$ be the set of all these $y$ 's. $Y$ is finite, and it is clear that the image of the restriction of any element of $\left[b_{1}, b_{2}\right]$ to $d\left(b_{1}\right) \cup d\left(b_{2}\right)$ (off which the element vanishes identically) is the same as the image of its restriction to $Y$. Thus, we need only find $c \in I=\left(b_{1}, b_{2}\right) \cap\left[b_{1}, b_{2}\right]$ such that $c$ does not vanish on $Y$. But this only means that $c \notin$ any of the primes in $\phi(Y)$. Since none of these primes contains $I$, neither can their union, and the result is proved.

6. Spaces with indeterminates. To complete our project of inverting Spec on subcategories of $\mathscr{S}$, we need only construct space-preserving functors from the subcategories to $\mathscr{B}$ such that the image objects are simple. For then we can apply $M$ followed by the (contravariant) forgetful functor from $\mathscr{B}$ to $\mathscr{C}$. We construct the functors to $\mathscr{B}$ in two steps, using an intermediate category $\mathscr{I}$ whose objects are spectral spaces together with indeterminates (the precise definition follows). In this section we define $\mathscr{I}$ and construct a space-preserving functor from $\mathscr{I}$ to $\mathscr{B}$ such that the image objects are simple. In the next section we obtain our goal by 
(quite easily) constructing space-preserving functors from various subcategories of $\mathscr{S}$ to $\mathscr{I}$. All our constructions are made over an arbitrary field $k$, which is fixed in this section and the next.

We shall assume that for each given set $E$ we are provided in a canonical way with a set $\{t(e)\}_{E}$, indexed by $E$ in one-one fashion, disjoint from $k$, which we shall always regard (as we may) as a family of algebraically independent indeterminates over $k$. (E.g. if our set theory precludes the membership of $k$ in a member of $k$, we can take $t(e)=(k, e)$. We shall not worry this point further.) Let $\mathscr{E}$ be the category of sets and functions, and let $Q$ be the (contravariant) functor from $\mathscr{S}$ to $\mathscr{E}$ which assigns to $X$ the set $Q(X)=\{U \subset X: U$ quasi-compact and open $\}$ and which assigns to $f$ the map $Q(f): U \mapsto f^{-1}(U)$.

Then we define a space with indeterminates to be a triple $I=(X, E, g)$, where $X$ is a spectral space, $E$ a set, and $g: E \rightarrow Q(X)$ a function such that $g(E)$ is an open sub-basis for $X$. In a hypothesis, $I$ will always represent $(X, E, g)$, with the analogous convention for $I^{\prime}$, etc. The spaces with indeterminates will be the objects of $\mathscr{I}$. A morphism from an object $I$ to an object $I^{\prime}$ of $\mathscr{I}$ will be a pair $(f, r)$, where $f$ is a spectral map from $X$ to $X^{\prime}$ and $r$ is an injective function from $E^{\prime}$ to $E$ such that $g^{\prime} r=Q(f) g$. We emphasize the injectivity of $r$ in this definition, for it is essential in the construction of the space-preserving functor from $\mathscr{I}$ to $\mathscr{B}$.

We now construct this functor. Let $I$ be a given object of $\mathscr{I}$. Let $K=k[t(e)]_{E}$. For each $e \in E$, let $T(e)$ be the element of $K^{X}$ (the ring of functions from $X$ to $K$ ) which has the value $t(e)$ on $g(e)$ and vanishes elsewhere. Let $A=k[T(e)]_{E} \subset K^{X}$. For each $x \in X$, let $A_{x}=k[t(e): e \in E$ and $x \in g(e)]=\{a(x): a \in A\}$. Let $A=$ $\left(X,\left\{A_{x}\right\}, A\right)$. Finally, if $p=(y, x) \in \sigma(X)$, define $v_{p}$ to be the unique valuation on $A_{y}^{*}$ satisfying (1) $v_{p}(t(e))=0$ or 1 , according as $x \in g(e)$ or not, and (2) if $s_{1}, \ldots, s_{n}$ are distinct monomials (products of $t(e)$ 's, or 1 ) in $A_{y}$ and $\lambda_{1}, \ldots, \lambda_{n} \in k-\{0\}$, then $v_{p}\left(\sum_{i} \lambda_{i} s_{i}\right)=\min _{i}\left\{v_{p}\left(s_{i}\right)\right\}$. It is easy to see that there is a unique such $v_{p}$ for each $p$ and moreover that it is nonnegative on $A_{y}$. It is quite straightforward to verify that $A, v$ is a simple indexed spring. We only note the following fact: let $\eta$ be the $k$-homomorphism from $K$ to $A$ which takes $t(e)$ to $T(e)$ for all $e$. Then $d\left(\prod_{\imath} T\left(e_{i}\right)\right)$ $=\bigcap_{i} g\left(e_{i}\right)$, and if $s_{1}, \ldots, s_{n}$ are distinct monomials in $K$ and $\lambda_{1}, \ldots, \lambda_{n} \in k-\{0\}$, then $d\left(\eta\left(\sum_{i} \lambda_{i} s_{i}\right)\right)=\bigcup_{i} d\left(\eta\left(s_{i}\right)\right)$.

For each $I$ in $\mathscr{I}$, let $H(I)$ be the indexed spring constructed in this way. $H$ will be the required functor; we must define it on morphisms. Let $(f, r)$ be a morphism in $\mathscr{I}$ from $I$ to $I^{\prime}$ and let $h_{0}$ be the unique $k$-homomorphism from $K_{I^{\prime}}$ to $K_{I}$ (where the indices have the obvious meaning) defined by $h_{0}: t(e) \rightarrow t(r(e))$. Then there is a unique $k$-homomorphism $h$ from $A_{I^{\prime}}$ to $A_{I}$ such that $h \eta=\eta h_{0}$, and $(f, h)$ is a morphism in $\mathscr{I}$ from $H(I)$ to $H\left(I^{\prime}\right)$. Once this is established, it is clear that we will have the required functor $H$.

We first show that for any $s \in K_{I}$, (\#) $f^{-1}(d(\eta(s)))=d\left(\eta\left(h_{0}(s)\right)\right)$. But this is obvious from the requirements on $r$ in the definition of a morphism in $\mathscr{I}$ (we need that $r$ be injective here). Since $\eta(s)=0 \Rightarrow d(\eta(s))=\varnothing \Rightarrow d\left(\eta\left(h_{0}(s)\right)\right)=\varnothing \Rightarrow \eta\left(h_{0}(s)\right)$ 
$=0$, there is a unique homomorphism $h$ as stated. That $(f, h)$ is a morphism in $\mathscr{B}$ is immediate from (\#). It is straightforward then to check that $(f, h)$ is a morphism in $\mathscr{I}$, because $h_{0}$ takes distinct monomials into distinct monomials (again, the injectivity of $r$ is needed), $h_{0}$ "preserves" products, and both $v$ 's convert products to corresponding sums, so that one can reduce to looking only at elements $T(e)$, for which the condition on the morphism for it to be in $\mathscr{I}$ is trivially verified. Summarizing:

THEOREM 5. $H$ is a space-preserving functor from $\mathscr{I}$ to $\mathscr{B}$ whose image objects are simple. If $F$ is the contravariant forgetful functor from $\mathscr{B}$ to $\mathscr{C}$, then $L=F M H$ is a space-preserving functor from $\mathscr{I}$ to $\mathscr{C}$.

\section{Inverting Spec.}

THEOREM 6. Every object and morphism in $\mathscr{S}$ is isomorphic to one in the image of Spec. Moreover, Spec is actually invertible on the following subcategories of $\mathscr{S}$ :

(a) The subcategory of all spectral spaces and surjective spectral maps.

(b) For each spectral space $X$, the subcategory of spectral subobjects of $X$ and inclusions of these.

(c) The full subcategory of $X$ whose objects are the $T_{1}$ spectral spaces.

Proof. We can prove the first statement by inverting Spec on the subcategory consisting of two spectral spaces $X, X^{\prime}$, the identity maps $1_{X}, 1_{X^{\prime}}$, and a given spectral map $f: X \rightarrow X^{\prime}$. (We assume $X \neq X^{\prime}$; if $X=X^{\prime}$, replace $X^{\prime}$ by a different, isomorphic, spectral space. This is to avoid considering powers of $f$.) To invert Spec on a subcategory, it is only necessary to define a space-preserving functor to $\mathscr{I}$. In this first case, define $G_{0}$ by $G_{0}(X)=\left(X, Q(X), 1_{Q(X)}\right), G_{0}\left(X^{\prime}\right)=\left(X^{\prime}, Q(X)\right.$ $\left.\times Q\left(X^{\prime}\right),(U, V) \mapsto V\right), G_{0}(1)=1$, and $G_{0}(f)=\left(f, U \mapsto\left(U, f^{-1}(U)\right)\right) . G_{0}$ is easily verified to be a space-preserving functor. For the other parts:

(a) For each $X$ let $G_{1}(X)=\left(X, Q(X), 1_{Q(X)}\right)$, and for each surjective $f$ let $G_{1}(f)$ $=(f, Q(f))$. (Here, $f$ surjective $\Rightarrow Q(f)$ injective.)

(b) For each $Y \subset X$ let $G_{2}(Y)=(Y, Q(X), U \mapsto U \cap Y)$, and for each $f$ let $G_{2}(f)=\left(f, 1_{Q(X)}\right)$.

(c) This is a much more elementary result which does not depend on Theorem 5. By the second corollary to Theorem 1, a $T_{1}$ spectral space is Hausdorff. In fact, this subcategory of $\mathscr{S}$ is actually the full subcategory of $\mathscr{T}$ whose objects are the totally disconnected compact (compact $\equiv$ quasi-compact Hausdorff) spaces. If $X$ is such a space, let $A(X)$ be the ring of continuous functions from $X$ to $k$, where $k$ has the discrete topology. It is easy to check that $(X,\{k\}, A(X))$ is a spring, and, using the fact that a totally disconnected compact space has a basis of open and closed sets [6, p. 20], that $\phi: X \rightarrow \operatorname{Spec} A(X)$ is surjective, i.e. that $X$ is isomorphic with Spec $A(X)$. We can easily see that $X \mapsto A(X)$, made into a functor in the obvious way, is the required space-preserving functor. 
This is the culmination of $\$ \S 2-7$. The following result shows that it is impossible to invert Spec on any very much larger subcategories of $\mathscr{S}$, so that Theorem 6 is fairly close to optimal.

Proposition 3. Spec is not invertible on these various kinds of subcategories of $\mathscr{S}$ :

(a) Subcategories containing a space $X$, a one-point space $P$, and maps $f: P \rightarrow X$, $f^{\prime}: X \rightarrow P$ such that $f(P)$ is not a closed point in $X$.

(b) Subcategories containing a space $Y$ with generic point, a family $\{X\}$ of spaces with generic point such that the cardinalities of the spaces in $\{X\}$ are not bounded, and for each $X \in\{X\}$ a map from $Y$ to $X$ (quite possibly, an embedding) which preserves generic points.

(c) Subcategories containing a one-point space $P$, spaces $X, Y$ with generic points, a map taking $P$ into the generic point of $X$, and two distinct maps from $Y$ to $X$ preserving generic points.

(d) For any spectral space $X$ containing a point $P$ with at least two points in its closure other than itself, the subcategory of spectral subobjects of $X$ and embeddings of these (as opposed to inclusions) in one another.

Proof. We may assume in each case that Spec has a right inverse $G$ taking values in the full subcategory $\mathscr{N}$ of $\mathscr{C}$ whose objects are the rings without radical (for the reduction functor is space-preserving), and look for a contradiction.

(a) $f^{\prime} f=1_{p} \Rightarrow G(f) G\left(f^{\prime}\right)=1_{G(P)}$. Then $G(f)$ must be a surjective homomorphism: $G(X) \rightarrow G(P)$, a field. Identifying $X$ with $\operatorname{Spec} G(X), P$ with $\operatorname{Spec} G(P)$, we see that $f$ is identified with Spec $G(f)$, and $f(P)$ must be a closed point.

(b) $f_{Y}: Y \rightarrow X$ preserves generic points $\Rightarrow G\left(f_{Y}\right)$ is an injection. But then rings of arbitrarily large cardinality are being injected into $G(Y)$.

(c) Let $f_{P}: P \rightarrow Y$ take $P$ to the generic point. Let $f, f^{\prime}: Y \rightarrow X$ preserve generic points, $f \neq f^{\prime} . f f_{P}=f^{\prime} f_{P} \Rightarrow G\left(f_{P}\right) G(f)=G\left(f_{P}\right) G\left(f^{\prime}\right)$. As in (b), $G\left(f_{P}\right)$ is injective. Hence, $G(f)=G\left(f^{\prime}\right)$. Applying Spec, $f=f^{\prime}$.

(d) We can reduce to the case where $X=\left\{P, P^{\prime}, P^{\prime \prime}\right\}$, where the three points are distinct and $P^{\prime}, P^{\prime \prime}$ are in $\mathrm{Cl} P$. Let $Y$ be the subobject $\left\{P, P^{\prime}\right\}$. We have $f: Y \rightarrow X$ by inclusion, $f^{\prime}: Y \rightarrow X$ by $P \mapsto P, P^{\prime} \mapsto P^{\prime \prime}$. Finally, we have $f_{P}: P \rightarrow Y$ by $P \mapsto P$. (c) now gives a contradiction.

8. Properties of spectral spaces. We collect here some interesting facts about spectral spaces. Observing that the notions of patch topology and patch (first paragraph of \$2) make sense whether the space considered is spectral or not, we extend them to arbitrary spaces.

Proposition 4. Let $X$ be a quasi-compact $T_{0}$ space which has a quasi-compact open basis that is closed under finite intersection. Then the following conditions are equivalent:

(i) $X$ is spectral.

(ii) Every nonempty irreducible closed subspace of $X$ has a generic point. 
(iii) Every family of quasi-compact open subsets of a closed subspace of $X$ with the finite intersection property has nonempty intersections.

(iv) $X$ with the patch topology is a compact space with a basis of open and closed sets.

(v) $X$ with the patch topology is quasi-compact.

(vi) A family of patches in $X$ with the finite intersection property has nonempty quasi-compact intersection.

Proof. Every implication in the chain (i) $\Leftrightarrow$ (ii) $\Rightarrow$ (v) $\Rightarrow$ (vi) $\Rightarrow$ (iv) $\Rightarrow$ (iii) $\Rightarrow$ (ii) is trivial, from the results of $\S 2$.

Now let us call a space quasi-Hausdorff if it satisfies the conclusion of the second corollary to Theorem 1 . Thus, the corollary asserts that spectral spaces are quasiHausdorff. This result has a partial converse, Proposition 6 below. First we observe that any $T_{0}$ space has a natural partial ordering, namely $y \leqq x$ if $x \in \mathrm{Cl} y$. (In Spec A, this means that $y \subset x$ as prime ideals.) Recall that a partially ordered set is lower directed if every finite subset has a lower bound.

Proposition 5. Every lower directed set $Y$ in a spectral space $X$ has a (unique) greatest lower bound $y$, and $y \in \mathrm{Cl} Y$.

Proof. $Y$ lower directed $\Rightarrow \mathrm{Cl} Y$ irreducible. It is easy to see that we may take $y$ to be the generic point of $\mathrm{Cl} Y$.

Proposition 6. $X$ as in Proposition 4 is spectral iff every closed subspace is quasiHausdorff and every lower directed set has a greatest lower bound in its closure.

Proof. $\Rightarrow$ is trivial. For $\Leftarrow$, we check that (ii) of Proposition 4 holds. $Y$ closed and irreducible $\Rightarrow Y$ quasi-Hausdorff. Together with irreducibility, this implies that $Y$ is lower directed. The greatest lower bound for $Y$ is then a generic point for $Y$.

We could make similar statements about upper directed sets. However, all such statements are immediate from the duality implicit in Proposition 8 below. We need an intermediate result first. It will be convenient to refer to $X$ with the patch topology as the patch space of $X$.

Proposition 7. Let $X$ be a compact space, and let $\{U\}$ be a family of open and closed (三compact open) subsets of $X$. Then $X$ with the topology which has $\{U\}$ as an open sub-basis is spectral iff it is $T_{0}$, in which case $X$ with its original topology is its patch space. Conversely, every spectral space arises from its patch space in this way.

Proof. Assume that $X$ with the weakened topology is $T_{0}$. It is easy to verify that it then satisfies the hypothesis of Proposition 4, using the fact that a space remains quasi-compact when its topology is weakened. We then need only show that the patch space of $X$ with the weakened topology is the original space, by condition (v) of Proposition 4. But the patch topology is Hausdorff and weaker than the original topology on $X$, which is compact; hence, they agree.

The converse part is trivial. 
Corollary. A space $X$ with a basis of quasi-compact open sets is spectral iff its patch space is compact.

Proof. We need only show $\Rightarrow$. $X$ must be $T_{0}$ because its patch space is. Now apply Proposition 7 to the patch space of $X$ with $\{U\}$ as the family of quasicompact open subsets of $X$ (which is a compact open sub-basis for the patch space of $X$ ).

We now obtain the topological duality mentioned in the introduction.

Proposition 8. Let $X$ be spectral. Retopologize $X$ by taking as a basis for the closed sets the quasi-compact open sets of $X$. Then $X$ with this new topology is spectral, and the new order induced on $X$ by this topology is precisely the reverse of the original order.

Proof. The patch space of $X$ with this new topology is clearly identical with the patch space of $X$ with the original topology, and $X$ in the new topology has a sub-basis for its open sets consisting of sets which are compact open in the patch topology. The new topology is clearly $T_{0}$, and it follows from Proposition 7 that $X$ with the new topology is spectral. The fact that the order is reversed is obvious.

9. The patch functor. If $X$ is spectral, let $T(X)$ be the patch space of $X$, and if $f: X \rightarrow X^{\prime}$ is spectral, let $T(f)$ be the corresponding map (same graph) from $T(X)$ to $T\left(X^{\prime}\right) . T$ is a covariant functor from $\mathscr{S}$ onto $\mathscr{K}$, the full subcategory of $\mathscr{T}$ whose objects are the totally disconnected compact spaces (equivalently, $\mathscr{K}$ is the full subcategory of $\mathscr{S}$ whose objects are the $T_{1}$ spectral spaces). $T$ is the "patch functor".

Let $J$ be the inclusion functor of $\mathscr{K}$ in $\mathscr{S} . T J$ is the identity functor on $\mathscr{K}$. Moreover, $T$ is adjoint to $J$ ( $J$ is coadjoint to $T$ ). This follows from the obvious one-one correspondence between spectral maps from $J(Y)$ to $X$ ( $Y, X$ given objects of $\mathscr{H}, \mathscr{S}$, respectively) and continuous ( $\Rightarrow$ spectral) maps from $Y$ to $T(X)$.

In the next section we show that this situation can be lifted, as it were, to $\mathscr{C}$. An interesting class of ring epimorphisms is brought to light. However, the adjointness of the functors is lost in the lifting.

10. A class of ring epimorphisms. Let $\mathscr{D}$ be the full subcategory of $\mathscr{C}$ whose objects are the rings without radical whose spectra are $T_{1}$ spaces, i.e. all of whose prime ideals are maximal. Up to isomorphism, Spec maps $\mathscr{D}$ onto $\mathscr{K}$. Let $J^{\prime}$ be the inclusion functor of $\mathscr{D}$ in $\mathscr{C} . J^{\prime}$ lifts $J$ in the sense that $\operatorname{Spec} J^{\prime} \cong J$ Spec, as functors. We want to define a functor from $\mathscr{C}$ to $\mathscr{D}$ which lifts $T$ similarly. Let $A$ be a ring with Spec $A=X$. For each $x \in X$, let $k_{x}=(A / x)^{*}$. Let $T^{\prime}(A)$ be the subring of $\prod_{x \in X} k_{x}$ consisting of elements $b$ such that for each $x \in X$ there is a patch neighborhood $U$ of $x$ and elements $a_{1}, a_{2}$ of $A$ such that for all $y \in U, a_{2}$ is not in $y$ (regarded as an ideal) and $b(y)=\psi_{y}\left(a_{1}\right) / \psi_{y}\left(a_{2}\right)$, where $\psi_{y}$ is the canonical homomorphism $A \rightarrow k_{y}$. It is a straightforward matter to verify that $T^{\prime}(A)$ is an object of $\mathscr{D}$ and that its spectrum may be identified in a natural way with $T(X)$. 
This brings us to the class of epimorphisms. The obvious homomorphism $h_{A}: A \rightarrow T^{\prime}(A)$ is an epimorphism, because $\operatorname{Spec} h_{A}$ (which may be identified with $\left.1_{X}: T(X) \rightarrow X\right)$ is injective, and the induced maps of local rings $\left(A_{x} \rightarrow k_{x}\right)$ are epimorphisms, in fact, surjections. These epimorphisms have been considered independently in [9]. Here, we only note the curious fact that in the case where $A$ is the integers, $h_{A}$ is an epimorphism from the integers to a ring which is not Noetherian.

We next observe that if $h: A \rightarrow B$ is a ring homomorphism, then there is a unique homomorphism $T^{\prime}(h): T^{\prime}(A) \rightarrow T^{\prime}(B)$ such that $T^{\prime}(h) h_{A}=h_{B} h$, and such that Spec $T^{\prime}(h)$ has the same graph as $\operatorname{Spec} h$ when $\operatorname{Spec} T^{\prime}(A)$, Spec $T^{\prime}(B)$ are identified with $T(\operatorname{Spec} A), T(\operatorname{Spec} B)$, respectively. This defines $T^{\prime}$ as a functor, and we then have $\operatorname{Spec} T^{\prime}=T$ Spec. Moreover, $T^{\prime} J^{\prime}$ is the identity on $\mathscr{D}$. This completes our discussion of the lifting.

\section{The category $\mathscr{S}$.}

THEOREM 7. Categorical products, images, arbitrary projective limits and finite coproducts exist in $\mathscr{S}$ and are identical with their counterparts in $\mathscr{T}$. Arbitrary coproducts and injective limits also exist in $\mathscr{S}$, as well as quotients; the last in the sense that given a spectral space $X$ and an equivalence relation $E$ on $X$ there is an essentially unique spectral surjection $g: X \rightarrow Y$ such that for each spectral map $f: X \rightarrow X^{\prime}$ which is constant on the equivalence classes of $E$ there is a unique spectral $h: Y \rightarrow X^{\prime}$ such that $f=h g$.

Proof. The first statement is easily verified. One need only show that the counterpart in $\mathscr{T}$ is spectral in each case. The details are omitted. To prove the existence of arbitrary injective limits, it suffices to prove that arbitrary coproducts and quotients exist. For quotients, let $D$ be the set of spectral maps from $X$ to a spectral space whose underlying set is a subset of $X$. Thus, we have at least one representative in $D$ of the isomorphism class of each surjective spectral map from $X$ to another spectral space. If $f \in D$, let $Y_{f}$ be the range space of $f$. Then it is easy to see that we may take $Y$ to be the image of $X$ under the evaluation map $g_{0}: X$ $\rightarrow \prod_{f \in D} Y_{f}$, and $g$ to be $g_{0}$ with its range restricted to $Y$.

It remains to show that arbitrary coproducts exist in $\mathscr{S}$. We refer to the results of the next section. The topological union of a family of spectral spaces is not spectral when the family is infinite, but is clearly "spectralifiable" in the sense of the next section, by Theorem 8 . From this it follows at once that arbitrary coproducts exist in $\mathscr{S}$.

12. Spectralifiable spaces. We now extend the notion of spectral map to a larger class of spaces. Call a space semispectral if the intersection of any two quasicompact open subsets is quasi-compact. Call an open subset $U$ of a topological space $X$ intersection quasi-compact open, or IQO, if for every quasi-compact open set $Q$ of $X, U \cap Q$ is quasi-compact. Thus, $X$ is semispectral iff the quasicompact open sets are IQO. Then a continuous map $f$ of semispectral spaces 
will be called spectral if $f^{-1}$ carries IQO sets to IQO sets. The semispectral spaces and spectral maps form a category, $\mathscr{U}$.

We note some useful facts. If the range space of a continuous function $f$ can be covered by quasi-compact open sets, then $f$ is spectral iff $f^{-1}$ carries quasi-compact open sets to IQO sets. If $X$ is quasi-compact, the IQO sets are simply the quasicompact open sets. Thus, $\mathscr{S}$ is a full subcategory of $\mathscr{U}$. Finally, observe that a finite union of IQO sets is IQO (even if the space is not semispectral) and that a finite intersection of IQO sets is IQO.

We now can make the notion of spectralifiable space precise. By a spectralification of a semispectral space $X$ we mean a spectral embedding $g$ of $X$ into a spectral space $X^{\prime}$ such that for every spectral space $Y$ and spectral map $f$ from $X$ to $Y$ there is a unique spectral map $f^{\prime}$ from $X^{\prime}$ to $Y$ such that $f=f^{\prime} g$. We call $X$ spectralifiable if it has a spectralification. Obviously, the spectralification is unique if it exists.

THEOREM 8. The following conditions on a space $X$ are equivalent:

(i) $X$ is spectralifiable.

(ii) $X$ is $T_{0}$ and the IQO sets are an open basis.

(iii) $X$ can be spectrally embedded in some spectral space.

Proof. (i) $\Rightarrow$ (iii) is obvious and (iii) $\Rightarrow$ (ii) is very easy, so that we need only show that (ii) $\Rightarrow$ (i). For this purpose we introduce a very simple spectral space $W$ which we shall continue to refer to in the next couple of sections. $W$ is the space whose underlying set is $\{0,1\}$ and whose open sets are precisely $\varnothing,\{0\},\{0,1\}$. Note that the ordering defined by the topology of $W$ is the usual one, $0<1$.

Let $V$ be the set of spectral maps from $X$ to $W$. Let $g_{0}: X \rightarrow W^{V}$ be the evaluation map. $W^{V}$ and $g_{0}$ are spectral. Let $X^{\prime}$ be the closure of $g_{0}(X)$ in $W^{V}$ in the patch topology, topologized, however, with the relative product topology from $W^{v}$, and let $g: X \rightarrow X^{\prime}$ be the restriction of $g_{0} . g$ is the required spectralification. The verification is detailed but straightforward. The condition that the IQO sets be a basis is needed so that the spectral maps will separate points from closed sets, which is precisely the condition which guarantees that $g_{0}$ will be an embedding [7, p. 116]. Given $f$, one defines $f^{\prime}$ by showing that for each $x^{\prime} \in X^{\prime}$ there is a unique point in $Y$ common to the patch closures in $Y$ of the sets of the form $f\left(v^{-1}\left(x_{v}^{\prime}\right)\right), v \in V$, where $x_{v}^{\prime}$ is the $v$ coordinate of $x^{\prime}$, and letting $f^{\prime}\left(x^{\prime}\right)$ be this point.

13. More characterizations of spectral spaces. As a corollary to the proof of Theorem 8 , we obtain the following result.

Proposition 9. $X$ is spectral iff it is homeomorphic with a patch in a product of copies on $W$.

Proposition 10. $X$ is spectral iff it is a projective limit of finite $T_{0}$ spaces.

Proof. Immediate from Proposition 9. 


\section{The maximal ideal space.}

Proposition 11. A space is homeomorphic with the maximal ideal space of some ring $A$ (with the topology inherited from Spec $A$ ), i.e. with the subspace of closed points of some spectral space iff the space is $T_{1}$ and quasi-compact.

Proof. $\Rightarrow$ is trivial. For the other half, suppose $M$ is $T_{1}$ and quasi-compact. Let $V$ be the set of continuous maps from $V$ to $W$. Let $f$ be the evaluation map (clearly, an embedding) from $M$ to $W^{v}$, and let $X$ be the closure of $f(M)$ in the patch topology on $W^{v}$, but give $X$ the relative product topology. The fact that $M$ is $T_{1}$ guarantees that every point of $f(M)$ is closed in $X$, while the fact that $M$ is quasicompact guarantees that every closed point in $X$ is actually in $M$.

We remark that if $M$ is Hausdorff as well, which means simply that it is compact, then it can be obtained very neatly as the maximal ideal space of the ring $C(M)$ of real-valued continuous functions on $M$. See [3, Chapter 4].

15. The order of the primes. We investigate which are the possible orders for the prime ideals of a ring, or, equivalently, for the points of a spectral space ordered in the standard way. We have observed (Proposition 8) that if an order occurs, so does its reverse. Some information can also be obtained from Theorem 7; e.g. if each of a family of orders can occur, then so can the product order on the cartesian product. Every finite order is possible, since every finite $T_{0}$ space is spectral.

We can characterize the possible orders as follows.

Proposition 12. Let $(X, \leqq)$ be an ordered set. Let $W=\{0,1\}$ as before. Then there is a spectral topology on $X$ inducing the given order $\leqq$ iff there is a family of order-preserving maps $V$ from $X$ to $W$ such that the following conditions hold:

(1) If $x, y \in X$ and $x \geq y$, then for some $v \in V, v(x)<v(y)$.

(2) If $h: V \rightarrow W$ and for each finite subset $V_{0}$ of $V$ there is an $x \in X$ such that $h(v)=v(x)$ for all $v \in V_{0}$, then there is an $x \in X$ such that for all $v \in V, h(v)=v(x)$.

Proof. For sufficiency, let $f$ be the evaluation map from $X$ to $W^{v}$, where $W$ is topologized as before. Then (1) guarantees that $f$ is injective, while (2) guarantees that the image is a patch. (1) then gives that the order on this patch agrees with that on $X$. Necessity is obvious.

The following result is then easily verified.

Proposition 13. A totally ordered set arises from a spectral topology iff the set is compact and totally disconnected in its order topology. In this case, there is only one spectral topology for the space inducing its order, and the corresponding patch topology is identical with the order topology on the set.

Along related lines, we ask to what extent the order induced by the topology determines the topology. Recall that a topological space is Noetherian if, equivalently, every strictly decreasing sequence of closed sets is finite, or if every open subspace is quasi-compact, or if every subspace is quasi-compact. (A Noetherian 
space is spectral iff it is $T_{0}$ and every nonempty irreducible closed subspace has a generic point.) Then:

Proposition 14. There is at most one Noetherian spectral topology inducing a given order on a space $X$.

Proof. It is well known that in a Noetherian space, a set is closed iff it is a finite union of irreducible closed subspaces, which means in this case (since the space is also spectral) a finite union of closures of points. Since the closure of a point is the set of points $\geqq$ it, the topology is determined by the order.

Proposition 15. Iff is a spectral map of spectral spaces and an order isomorphism, then $f$ is a homeomorphism.

Proof. As a map of patch spaces, which are compact, $f$ is a continuous bijection, hence, a homeomorphism. Since a subset of a spectral space is closed iff it is a patch and contains the closure of each of its points, the result is obvious.

16. The underlying spaces of preschemes and schemes. We call a space locally spectral if it has a cover by open spectral subspaces. Note that such a space must be $T_{0}$.

THEOREM 9. The locally spectral spaces are precisely the underlying spaces of preschemes.

Proof. It is trivial that the underlying space of a prescheme is locally spectral. Let $X$ be a locally spectral space. We want to construct a prescheme having $X$ as its underlying space. To do this, we first observe that the open spectral subspaces of $X$ form an open basis $B$. We shall construct a presheaf of rings on $X$ with respect to $B$. First, fix a field $k$ as in $\S 6$. Let $\mathscr{R}$ be the category of open spectral subspaces of $X$ and inclusions of these in one another. We now define a space-preserving functor $G$ from $\mathscr{R}$ to $\mathscr{I}$. For each $Y$ in $R$ let $E(Y)$ be the set of objects of $\mathscr{R}$ whose intersections with $Y$ are also in $\mathscr{R}$, and let $G(Y)=(Y, E(Y), U \mapsto U \cap Y)$. Note that $Q(Y) \subset E(Y)$; in fact, the map $U \mapsto U \cap Y$ is a retraction. If $Y \subset Y^{\prime}$, then $E\left(Y^{\prime}\right) \subset E(Y)$, so that the inclusion $f: Y \rightarrow Y^{\prime}$ induces an injection $j_{f}: E\left(Y^{\prime}\right)$ $\rightarrow E(Y)$. We define $G(f)=\left(f, j_{f}\right)$. Now, if $L=F M H$ is the functor of Theorem 5 , then $L G: \mathscr{R} \rightarrow \mathscr{C}$. But giving a functor from $\mathscr{R}$ to $\mathscr{C}$ is precisely the same as giving a presheaf on $X$ with respect to $B$. Let $\mathcal{O}_{X}$ be the associated sheaf of $L G$. We shall show that $\left(X, \mathcal{O}_{X}\right)$ is a prescheme. In fact, we shall show that for each open spectral subspace $Y$ of $X,\left(Y, \mathcal{O}_{X \mid Y}\right)$ is an affine scheme.

Let $\Omega=k(\{t(U): U \in B\})$ (notation as in $\S 6$ ). We note that from the constructions of $G, H$ and $M$, for each open set $V \subset X, \Gamma\left(V, \mathcal{O}_{X}\right)$ may be regarded as a ring of functions from $V$ to the field $\Omega$, and that the sheaf homomorphisms $\Gamma\left(V, \mathcal{O}_{X}\right)$ $\rightarrow \Gamma\left(W, \mathcal{O}_{X}\right), W \subset V$, are ordinary restriction homomorphisms. If $a \in \Omega^{V}$, then $a \in \Gamma\left(V, \mathcal{O}_{X}\right)$ iff there is an open spectral cover $\left\{V_{i}\right\}$ of $V$ such that for each $i$ the 
restriction of $a$ to $V_{i}$ is in $L G\left(V_{i}\right)$. If $V$ is quasi-compact, $\left\{V_{i}\right\}$ may be taken to be finite. We make the convention that an element of $\Gamma\left(V, \mathcal{O}_{X}\right)$ is extended (as a function) to a larger set $V^{\prime}$ by letting it be zero off $V$. Of course, this extended iunction need not belong to $\Gamma\left(V^{\prime}, \mathcal{O}_{X}\right)$.

For each $y \in Y$, let $\Gamma_{y}=\left\{a(y): a \in \Gamma\left(Y, \mathcal{O}_{X}\right)\right\}$. Then $\left(Y,\left\{\Gamma_{y}\right\}, \Gamma\left(Y, \mathcal{O}_{X}\right)\right)$ is easily seen to be a spring. Moreover, this spring is obviously simple. Finally, suppose $a$, $a^{\prime} \in \Gamma\left(Y, \mathcal{O}_{X}\right)$ and $z\left(a^{\prime}\right) \subset z(a)$. Choosing a suitably fine quasi-compact open covering $\left\{Y_{i}\right\}$ of $Y$ (and using the subscript $i$ to indicate restriction to $Y_{i}$ ), we can assume that $a_{i}, a_{i}^{\prime} \in L G\left(Y_{i}\right)$, and that $z\left(a_{i}^{\prime}\right) \subset z\left(a_{i}\right)$, for all $i$. Hence, for each $i$ we can choose $N_{i}$ such that $a_{i}^{n} \# a_{i}^{\prime} \in L G\left(Y_{i}\right)$ whenever $n \geqq N_{i}$ and choosing $n \geqq \max _{i}\left\{N_{i}\right\}$ we have $a_{i}^{n} \# a_{i}^{\prime} \in L G\left(Y_{i}\right)$, for all $i$. Then $a^{n} \# a^{\prime} \in \Gamma\left(Y, \mathcal{O}_{X}\right)$, and $a \in R\left(a^{\prime}\right)$ in this ring. Thus, $\left.{ }^{* *}\right)$ holds and the spring $\left(Y,\left\{\Gamma_{y}\right\}, \Gamma\left(Y, \mathcal{O}_{X}\right)\right)$ is affine.

Thus, we may identify $Y$ with $\operatorname{Spec} \Gamma\left(Y, \mathcal{O}_{X}\right)$ and use the notations $z$ and $Z$ (also $d$ and $D$ ) interchangeably. Now let $0 \neq b \in \Gamma\left(Y, \mathcal{O}_{X}\right)$ be arbitrary. There is an obvious homomorphism $S^{-1} \Gamma\left(Y, \mathcal{O}_{X}\right) \rightarrow \Gamma\left(D(b), \mathcal{O}_{X}\right)$, where $S$ is the multiplicative system generated by $b$. To complete the proof that $\left(Y, \mathcal{O}_{X \mid Y}\right)$ is affine, we need only show that all these homomorphisms (for various $b$ ) are isomorphisms. Injectivity is trivial. To show surjectivity, let $a \in \Gamma\left(D(b), \mathcal{O}_{X}\right)$. Then we need only prove that $b^{n} a \in \Gamma\left(Y, \mathcal{O}_{X}\right)$ for sufficiently large $n$. Choose a finite quasi-compact open covering $\left\{D_{i}\right\}$ of $D(b)$ such that $a_{i} \in L G\left(D_{i}\right)$. For each $D_{i}$ choose $b_{i} \in L G(Y)$ such that $D_{i}=D\left(b_{i}\right)$. Suppose we can show that for each $i, b_{i}^{n} a_{i} \in \Gamma\left(Y, \mathcal{O}_{X}\right)$ for suitable $n$. Let $c_{i}=b_{i}^{n}$ for this $n$. Then we have found elements $c_{i}$ of $\Gamma\left(Y, \mathcal{O}_{X}\right)$ such that $c_{i} a_{i}=\dot{c}_{i} a \in \Gamma\left(Y, \mathcal{O}_{X}\right)$, for each $i$, and $\bigcap_{i} Z\left(c_{i}\right)=Z(b)$. Since $\Gamma\left(Y, \mathcal{O}_{X}\right)$ is simple when regarded as an affine spring, it follows from the proof of Theorem 4 that there is an element $c$ of the ideal $\left(c_{i}\right)$ generated by the $c_{i}$ in $\Gamma\left(Y, \mathcal{O}_{X}\right)$ such that $Z(c)=\bigcap_{i} Z\left(c_{i}\right)$. Then $c a \in \Gamma\left(Y, \mathcal{O}_{X}\right)$ and $Z(c)=Z(b)$, so that for some $n, b^{n} \in(c)$ $\Rightarrow b^{n} a \in \Gamma\left(Y, \mathcal{O}_{X}\right)$.

It remains to show that if $b_{i} \in L G(Y)$ and $a_{i} \in L G\left(D\left(b_{i}\right)\right)$, then for some $n$, $b_{i}^{n} a_{i} \in \Gamma\left(Y, \mathcal{O}_{X}\right)$. In fact, we shall show that for suitable $n, b_{i}^{n} a_{i} \in L G(Y)$. We drop the subscript $i$. Let $A=\left\{a \in L G(D(b))\right.$ : for some $\left.n, b^{n} a \in L G(Y)\right\}$. Then what we want to show is that $A=L G(D(b))$. $A$ is a subring of $L G(D(b))$, and contains the underlying ring of $H G(D(b))$ (for these elements, we can take $n=0$ ). If we can show that whenever $a, a^{\prime} \in A$ and $a \# a^{\prime} \in L G(D(b))$ then $a \# a^{\prime} \in A$, the result will follow from the definition of $M$. Hence suppose $b^{n} a, b^{n} a^{\prime} \in L G(Y)$. Looking at $D(b)$ and $Z(b)$ separately, we see that $b^{m}\left(a \# a^{\prime}\right)=b^{m+n} a \# b^{n} a^{\prime}$. Thus, it suffices to show that condition (i) of Theorem 3 holds for the pair $\left(b^{m+n} a, b^{n} a^{\prime}\right)$ when $m$ is sufficiently large. Choose $m>\max _{p}\left\{v_{p}\left(b^{n} a^{\prime}\right)\right\}$. One then checks the cases $(y, x) \in D(b) \times D(b)$, $(y, x) \in D(b) \times Z(b)$ separately. Note that if $y \in Z(b), x \in \mathrm{Cl} y$ must also. In the first case, one uses the fact that $a \# a^{\prime} \in L G(D(b))$. In the second case, one uses the size of $m$ to get condition (i) to hold. (If $(y, x) \in Z(b) \times Z(b)$ there is nothing to check.)

It is much simpler to characterize the underlying spaces of schemes. 
Proposition 16. The following conditions on a topological space $X$ are equivalent.

(i) $X$ is the underlying space of some scheme.

(ii) $X$ is locally spectral and semispectral.

(iii) $X$ is homeomorphic with an open subspace of a spectral space.

(iv) $X$ is the underlying space of an open subscheme of an affine scheme.

Proof. (iii) $\Rightarrow$ (iv) $\Rightarrow$ (i) $\Rightarrow$ (ii) is trivial (the underlying space of a scheme is semispectral because in a scheme, the intersection of two open affines is affine [2, p. 74], and every quasi-compact open set is a finite union of open affines). To show that (ii) $\Rightarrow$ (iii), we consider the embedding $X \rightarrow W^{v}$ used in the proof of Theorem 8 . The additional hypothesis that $X$ be locally spectral is precisely what is needed to guarantee that the (topological) image of $X$ in $W^{v}$ be open.

\section{REFERENCES}

1. N. Bourbaki, Algèbre commutative, Chapitre 2, Hermann, Paris, 1961.

2. J. Dieudonné, Algebraic geometry, Lecture Notes, Univ. of Maryland, College Park, 1962.

3. L. Gillman and M. Jerison, Rings of continuous functions, Van Nostrand, Princeton, N. J., 1960.

4. M. Hochster, Prime ideal structure in commutative rings, Thesis, Princeton Univ., Princeton, N. J., 1967.

5. - Symbolic powers in Noetherian domains (to appear).

6. W. Hurewicz and H. Wallman, Dimension theory, Princeton Univ. Press, Princeton, N. J., 1941.

7. J. L. Kelley, General topology, Van Nostrand, Princeton, N. J., 1955.

8. R. G. Montgomery, $\operatorname{Spec} A$ as a set with algebraic structure, Abstract $68 \mathrm{~T}-458$, Notices Amer. Math. Soc. 15 (1968), 637.

9. J.-P. Olivier, Anneaux absolument plats universels et epimorphismes d'anneaux, C. R. Acad. Sci. Paris Ser. A et B 266 (1968), 317.

Princeton University, Princeton, NeW Jersey

UNIVERSITY OF MINNESOTA, Minneapolis, Minnesota 